\title{
Schnelle Diagnose in kritischen Situationen: Wie die Radiologie Frühgeborenen hilft
}

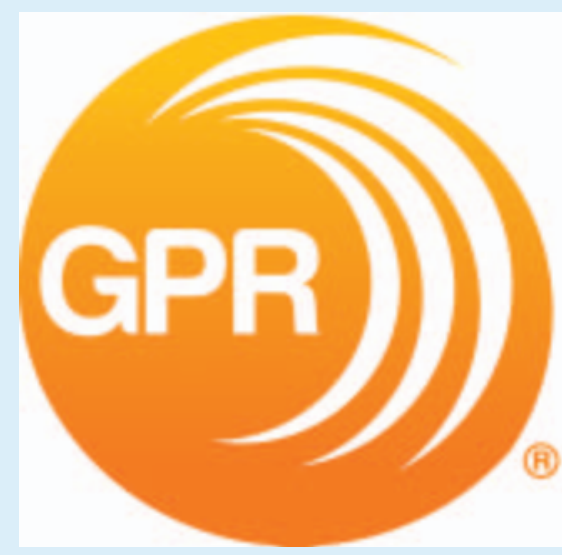

Sie wiegen manchmal nur 300 Gramm und leiden an ganz eigenen Krankheiten: Frühgeborene starten mit vielen Risiken ins Leben. Eine besondere Herausforderung für Eltern und Ärztinnen sowie Ärzte. Spezialisierte Kinderradiologinnen und Kinderradiologen helfen, Erkrankungen schnell zu erkennen und den Weg für die richtige Behandlung zu ebnen. Anlässlich des Weltfrühgeborenentags am 17. November hatten wir mit der Kinderradiologin Dr. Stephanie Spieth vom Universitätsklinikum Carl Gustav Carus in Dresden gesprochen.

Frau Dr. Spieth, viele Frühgeborene haben aufgrund ihrer teilweise extremen Unreife gesundheitliche Komplikationen. Was begegnet Ihnen als Kinderradiologin am häufigsten?

Die unreifen Organsysteme sind besonders anfällig für viele Erkrankungen. In der Kinderradiologie sehen wir vor allem pulmonale, abdominale und zentralnervöse Komplikationen. Typische Lungenerkrankungen sind anfangs das Atemnotsyndrom und später die bronchopulmonale Dysplasie, eine chronische Lungenerkrankung. Abdominal ist die nekrotisierende Enterokolitis, eine schwere Entzündung der Darmwand, besonders gefürchtet. Intrakraniell sind Sauerstoffmangel und Blutungen für die häufigsten Komplikationen verantwortlich. Hierzu zählen Schädigungen der weißen Gehirnsubstanz wie die periventrikuläre Leukomalazie, intraventrikuläre Blutungen oder periventrikuläre hämorrhagische Infarzierungen. Die Blutungen können zu Liquorzir- kulationsstörungen und konsekutiv zu einem Hydrozephalus führen. Im weiteren Verlauf kann es bei den beschriebenen Erkrankungen zu einer Beeinträchtigung der neurologischen Entwicklung kommen.

Wie kann da die Radiologie jeweils weiterhelfen?

Mittels Bildgebung kann die Diagnose frühzeitig gestellt, die Ausprägung beurteilt und der Interventionsbedarf eingeschätzt werden. Hierfür kommen bei den Frühgeborenen vor allem der Ultraschall bei Schädel- und Abdomenuntersuchungen sowie das Röntgen bei Thorax- und Abdomenuntersuchungen zum Einsatz, seltener die MRT im Schädelbereich.

Die Untersuchung so kleiner Patientinnen und Patienten ist eine große Herausforderung. Auf welche Punkte müssen Sie als Kinderradiologin besonders achten?

Eine besonders strenge Indikationsstellung ist für uns sehr wichtig, das bedeutet, es muss eine kritische Situation vorliegen, die einer weiteren Abklärung bedarf. Denn jede Untersuchung bedeutet Stress für das Frühgeborene und stellt ein gewisses Risiko dar, etwa durch Umlagerung mit Dislokationen oder Wärmeverlust. Dem kann unter anderem durch die Untersuchung im Inkubator entgegengewirkt werden. Bisher erfolgen der Ultraschall und die Röntgenuntersuchung am Bett, in Zukunft wird das auch mit der MRT möglich sein. Bis dahin werden Transportinkubatoren und mancherorts auch MR-Inkubatoren zum konstanten Halten der Temperatur eingesetzt.

\section{Braucht es für die Untersuchung von Frühgeborenen besondere Kenntnisse?}

Auf jeden Fall, sowohl auf Seite der Medizinisch-Technischen Radiologieassistentinnen und -asisstenten als auch ärztlicherseits, damit die Untersuchung kindgerecht durchgeführt und korrekt interpretiert werden kann. Technisch steht hier bei der Röntgenuntersuchung vor allem der Strahlenschutz im Vordergrund, bei der MRT-Untersuchung ist die geringe Patientengröße

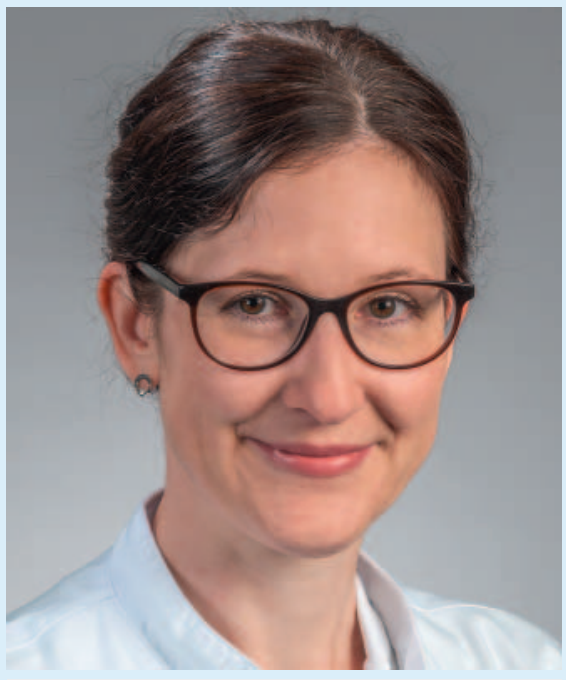

Dr. Stephanie Spieth

eine Herausforderung. So ist ein Frühgeborenes der 24. Schwangerschaftswoche nur etwa $30 \mathrm{~cm}$ lang. Zudem haben wir es bei den Frühgeborenen nicht nur mit anderen anatomischen beziehungsweise physiologischen Verhältnissen, sondern auch mit Frühchen-spezifischen Krankheitsbildern zu tun.

\section{Warum ist der Strahlenschutz bei Röntgenuntersuchungen so wichtig?}

Aufgrund der besonders hohen Strahlenempfindlichkeit von kleinen Kindern im Vergleich zu Erwachsenen, unter anderem durch Unterschiede in Anatomie und Physiologie, muss die Anwendung ionisierender Strahlung sehr bedacht erfolgen. Zudem ist oft umso häufiger eine Bildgebung erforderlich, je geringer das vorliegende Gestationsalter ist.

\section{Gibt es auch bei Ultraschall und MRT Sicherheitsaspekte, die Sie beachten?}

Hier stellt eher, falls notwendig, der innerklinische Transport mit den bereits genannten Risiken der Stressinduktion durch unter anderem Umlagern oder Wärmeverlust sowie die notwendige Überwachung ein Problem dar. Für die MRT-Untersuchung gelten ansonsten die gleichen Sicherheitsvorkehrungen wie für größere Kinder und Erwachsene auch. 


\section{Können Eltern bei den Untersuchungen dabei sein? Und von wem erfahren sie das Ergebnis der Untersuchung?}

Prinzipiell können die Eltern bei den Untersuchungen mit dabei sein, wobei das eher bei den Untersuchungen am Bett der Fall ist. Die MRT-Untersuchung erfolgt in der Regel in Begleitung der neonatologischen Kollegen, die den Eltern meist auch den Befund übermitteln. Besonders wichtig ist deshalb die enge Zusammenarbeit der Kinderradiologen mit den pädiatrischen Intensivmedizinern, auch im Vorfeld der Untersuchung. Hier steht die gemeinsame Aufklärung der Eltern im Vordergrund, um ihnen die Notwendigkeit der Untersuchung zu vermitteln und ihnen die Angst davor zu nehmen.

\section{Vielen Dank für das Gespräch, Frau Dr. Spieth!}

\section{Was ist Kinderradiologie?}

Die Kinder- und Jugendradiologie (offizielle Bezeichnung durch die Bundesärztekammer seit 2019) beschäftigt sich mit der speziellen Bildgebung beim Neugeborenen, Säugling, Kleinkind, Schulkind und Jugendlichen.

Kinderradiologinnen und Kinderradiologen sind Radiologinnen und Radiologen, die in einer (in Deutschland aktuell zweijährigen) fachärztlichen Schwerpunktweiterbildung speziell in der Kinderradiologie geschult sind. Sie legen großen Wert darauf, die Un- tersuchungen von Kindern schonend und mit geringstmöglicher Strahlendosis durchzuführen. Bevorzugte Methoden kinderradiologischer Bildgebung sind daher die ohne Röntgenstrahlen arbeitenden Verfahren wie die Sonografie (Ultraschalluntersuchung) und die Magnetresonanztomografie (MRT).

Die GPR als wissenschaftliche Vereinigung der deutschsprachigen Kinderradiologen (Deutschland, Österreich und Schweiz) wurde 1963 gegründet. Aktuell zählt sie über 380 Mitglieder. Im Fokus steht die wissenschaftliche Vernetzung, die Nachwuchsförderung, die Vertretung des Fachs im Wissenschaftsbetrieb und in der Berufspolitik.

\section{Save the Date}

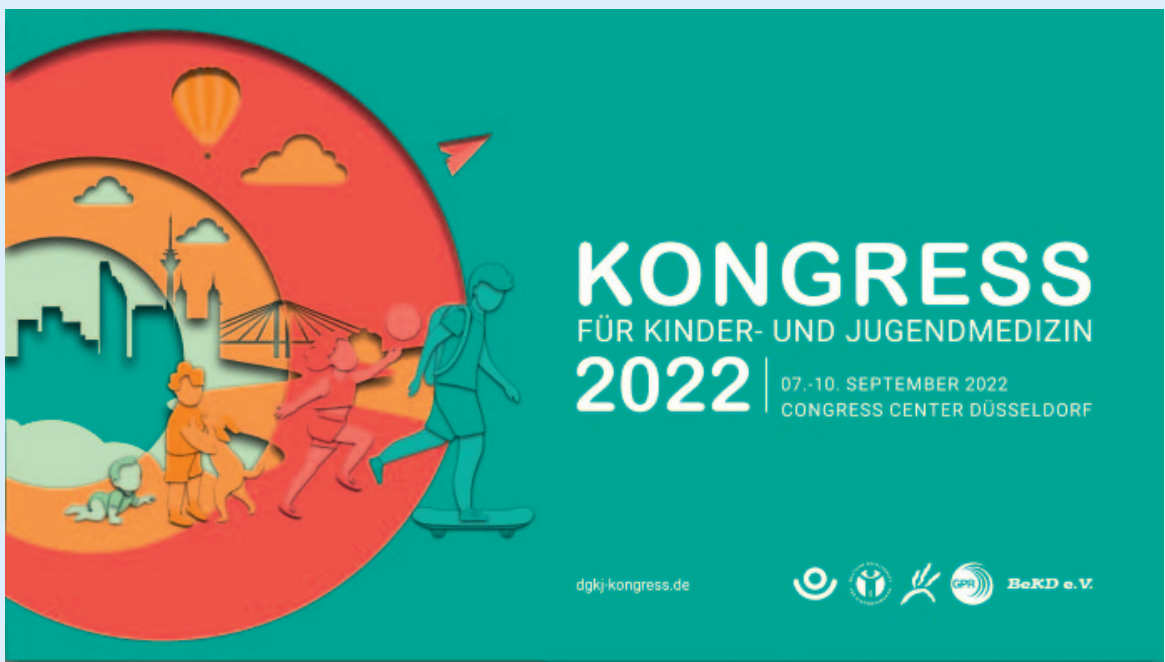

